\title{
World Journal of Pediatric heart surgery in China:
Pediatric Surgery progress and challenges
}

Xumin Mo, ${ }^{1}$ Di Yu, ${ }^{1}$ Qiang Shu ${ }^{\odot 2}$

To cite: Mo X, Yu D, Shu Q. Pediatric heart surgery in China: progress and challenges. World Jnl Ped Surgery 2019;2:e000048 doi:10.1136/wjps-2019-000048

Received 8 March 2019 Accepted 15 March 2019

Check for updates

(c) Author(s) (or their employer(s)) 2019. Re-use permitted under CC BY-NC. No commercial re-use. See rights and permissions. Published by BMJ.

'Department of Cardiothoracic Surgery, Children's Hospital of Nanjing Medical University, Najing, China

${ }^{2}$ Department of Cardiothoracic Surgery, Children's Hospital, Zhejiang University School of Medicine, Hangzhou, China

Correspondence to Dr Qiang Shu,Hangzhou, China; wjps@zju.edu.cn
Congenital heart disease (CHD) has become the most common birth defect and is now the first leading cause of infant mortality due to congenital malformations in China. According to the 2012 Birth Defects Report, CHD has been the first leading birth defect in China since a decade ago. Patients with CHD have reached 2 million in China, and newly diagnosed patients were estimated to be 150 000-2 00000 annually. ${ }^{1}$

China has the largest population in the world and with $15 \%$ of all children worldwide. In 2016, there were 17.9 million newborns. ${ }^{2}$ After adoption of the universal two-child policy since 2015, the incidence of CHD may be increased as a result of high portion of women with high maternal age. Though with such high incidence of CHD and large population of patients with CHD, no country-level data registry has been established. Current epidemiology of CHD is regional and not accurate, lacking high evidence. According to the available data, we think that the incidence of CHD is varied across the country. The data of 2011 showed that incidence of CHD diagnosed during perinatal period is $6.8-14.39 \%$. Some regional surveillance data showed that the prevalence of CHD was $3.81 \%$ in Jiuquan City from 2002 to 2003, 8.04\% in Beijing City during 2007-2012, and 9.04\% in Guangdong Province during 2006-2015. ${ }^{3}$ Concerning the disease types, ventricular septal defect, atrial septal defect, and patent ductus arteriosus account for $75 \%-80 \%$ of all CHD. The nationwide epidemiological study on CHD remains difficult in China because of no unified diagnosis techniques and guidelines among different regions, no standard surveillance in the migration population, and lacking knowledge of collecting big data for analysis in the population. A delightful news is that since 2018, the Chinese Government has launched national neonatal screening of congenital heart disease, which may help to investigate the incidence of congenital heart disease in China.

\section{CURRENT STATUS OF CONGENITAL HEART SURGERIES IN CHINA}

Healthcare resources for CHD

China has 700 pediatric cardiac centers which can operate on children with CHD in China. Most of these centers are located in the municipalities, provincial cities, and in economically developed coastal cities. Fuwai Hospital in Beijing has the highest surgical volume per year, and Shanghai Children's Medical Center is in the leading position among all children's hospitals around Asia. In 2015, the number of operations in the top 10 hospitals accounted for $30.9 \%$ of all surgeries. According to Heart Surgery and Extracorporeal Circulation Data $2016^{3}$ White Paper, with 76256 pediatric cardiac operations in 2016, the surgical volume was 2604 fewer than that in 2015. ${ }^{3}$ From 2011 to 2015 , the total pediatric cardiac operations and extracorporeal circulation volume increased remarkably (figure 1). However, overall surgical volume under extracorporeal circulation increment was very slow because of application of less invasive catheterization and hybrid procedures. The ratio of employing extracorporeal circulation for pediatric cardiac operations reduced since 2014 and reduced to be less than $80 \%$ for the first time in $2016 .^{3}$

Data showed the pediatric cardiac surgical volume decreased continuously even after a universal adoption of two-child policy in 2015. It is a worldwide trend that live births of CHD decreased, which may be partly due to improved maternal healthcare and intense prenatal diagnosis. A survey was carried out in 39 children's hospitals and women's and children's hospitals from 2012 to 2016; the results revealed that the total 5 -year rate of operation volume increased by $18.25 \%$; however, with a gradual reduction in operation volume in the later 3 years, the total number of extracorporeal circulation increased by $14.51 \%$, but reduced by $2.68 \%$ in 2016 . The number of extracorporeal circulation reduced in the later 3 years in a succession (2014-2016). The data suggest that the volume of CHD operations is reducing accordingly. 


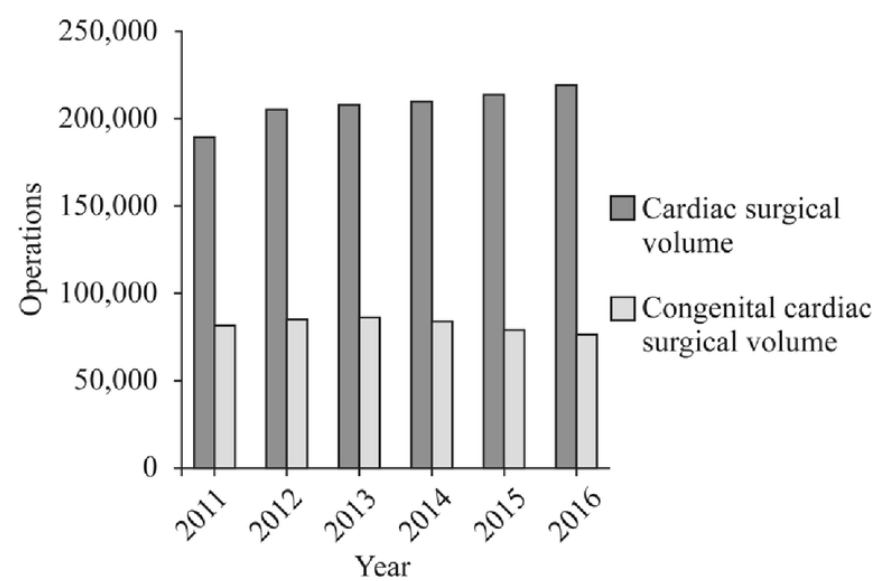

Figure 1 Comparison of total cardiac surgical volume and congenital cardiac surgical volume from 2011 to 2016

\section{Healthcare expenses for CHD}

Most parents will take their children to a large tertiary-level hospital among the local CHD Diagnosis and Treatment Network or to children's hospitals with pediatric cardiac centers. Due to the change of medical insurance policy in the past decade, no unified standards can be referred for calculation of the treatment cost of CHD. There are great disparities in the treatment cost among different regions and during different periods. In the Beijing City, the in-hospital cost for treating a simple CHD increased from 24800 RMB in 2005 to $28700 \mathrm{RMB}$ in 2010, and the cost was 32 038 RMB and 30370 RMB in Shanghai City (2004) and Guangdong Province (2012-2013), respectively. According to the data of the recent 2 years, the national-level mean treatment cost for CHD is about $46000 \mathrm{RMB}$. And the cost is different concerning different types of disease. For example, the mean treatment cost for Tetralogy of Fallot (TOF) was as high of $46000 \mathrm{RMB}$, and the cost for reoperation was $77500 \mathrm{RMB}$, which is far higher than those of a single operation. Taking into account of economical losses due to parents' reduced working time, the gap will become much greater.

We should attach more importance to those patients from underprivileged families and Middle-Western areas with low economical level in China. Treatment for congenital heart disease may be still out of reach to these children because of high expense of treatment, poor access to healthcare, underdeveloped health systems, etc. As shown in the 2010 report of Pilot Work of Increasing the Medical Care Level of Major Diseases for Rural Residents by the Ministry of Health, new rural cooperative medical schemes (NRCMS) should increase the reimbursement of medical insurance to at least $70 \%$ for children with CHD. For those patients meeting the Medical Support policy, an additional $>20 \%$ of treatment cost should be reimbursed by NRCMS.

Due to the different medical insurance policy among regions, the rate of the reimbursement of medical insurance varied greatly. Economical level of local regions impact significantly on the reimbursement rate of medical insurance. The reimbursement rate is much higher in the cities and provinces with high economical level. In some regions, the reimbursement rate for CHD is as high as $100 \%$ and as low as $30 \%$ in the underdeveloped regions. Hopefully, except the government-leading medical insurance support, there are now a lot of non-profit charity organizations supporting CHD treatment. These charity organizations have provided strong economical support for those underprivileged families; however, some families lack information of these charity organizations and policies, which should be highlighted in the future CHD management.

\section{Status of the congenital cardiac specialty in China}

In the recent 10 years, the specialty of pediatric cardiac surgery has achieved staggering developments in China. The progress is embodied by increased success rate of surgeries and steadily growing cardiac surgeons team. We also have made a great progress in treating severe complex CHD including TOF, pulmonary atresia and ventricular septal defect, coarctation of the aorta, etc. These complex CHD procedures have been established in pediatric cardiac centers. There are many innovative techniques being started by cardiac surgeons in China including aortic double conical transposition, tricuspid valve subtraction angioplasty, and one-stop hybrid procedure. Several international leading procedures including slide surgery for tracheal atresia, newborn cardiac surgery, fetal cardiac surgery, etc, have achieved a favorable progress in China. With the development of techniques, indications for complex procedure have been expanded using more advanced techniques, instruments, and drugs. Many staged procedures have been changed to one-stage procedure, and we are making possible the impossible. Accordingly, post-surgery complication rate, duration of hospitalization, and intensive care unit stay reduced gradually, and in some large pediatric cardiac centers, the level of surgery is approaching to the most leading level worldwide and even being in the leading level in some CHD procedures.

With advances of catheter techniques, catheter-based therapy for CHD has been introduced into many centers in China. In 21 January 2006, the first case of minimal invasive occlusion for repairing ventricular septal defect was performed in Nanjing Children's Hospital. Since then, minimal invasive procedures for CHD including transthoracic occlusion, transcatheter closure under the guidance of transthoracic echocardiography, percutaneous transthoracic occlusion have achieved rapid developments in China. CHD surgery teams in China have also made prominent innovative contributions in establishing minimally invasive technology and occlusion techniques, designing and improving medical devices such as occluder, etc. The staggering development of minimally invasive procedures for CHD in China has become a global-calling card of pediatric cardiac surgery specialty. The wide application of minimally invasive procedures are the result of patients' requirements. The application 
of hybrid procedures facilitate surgery to be less invasive and more safer than the traditional surgery.

The past 5 years witnessed a change in the trend in the advancement of pediatric cardiac surgery specialty, disease types, and techniques in CHD. The advancement of specialty can be seen widely in the adoption of prenatal diagnosis integration, advancing optimal surgery time, increased number of infants and young children treated in children's hospitals, and reduced disparity in surgery techniques between western undeveloped and coastal developed regions in China. Concerning the disease type changes, malformations of left heart system decreased and total anomalous pulmonary venous connection increased. Cases of preterm infants and babies born after fertility treatment increased. New characteristics of techniques include wide adoption of supporting techniques (like extracorporeal membrane oxygenation, continuous renal replacement therapy, peripherally inserted central catheter, bronchofiberscope, and stents) and implementing of associated surgery techniques (slide tracheoplasty, cardiac pneumothorax homochronous operation).

\section{Challenges of the Chinese pediatric cardiac surgery specialty}

The Chinese congenital heart surgery specialty is facing many challenges. First, the characteristics of cases change to be of much younger age, complex type, under emergency critical conditions. Second, wide adoption of minimally invasive techniques requiring restrict counsel of indications, large sample long-term follow-up, multiple-disciplinary collaboration, and building specialized CHD diagnosis and treatment team; for complex CHD, innovation procedures and optimization are important for increasing the patients' life quality. Third, challenges come from biomaterials, and application of bioabsorbable stents will change the clinical practice. Fourth, challenges from internet techniques and the application of of artificial intelligence in diagnosing CHD. Fifth, ethical challenges from improved fetal diagnosis of CHD. Sixth, the particular challenges with Chinese characteristics. With the decreasing trend of total CHD cases in China, patient's regional geographical distribution is changing. Not all those CHD children from Western China have to seek therapy to developed cities. The disparities among regions become less significant; CHD volume in general hospitals decreased and instead with an increasing surgical volume in children's hospitals. In future, most patients with CHD will be treated at large-scale provincial hospitals, and medical insurance out-of-town payment system should be strengthened.

\section{Future development directions}

The major five future development goals of China CHD specialty are as follows: (1) specialty construction: independent construction of specialty in general and children's hospitals, construction of specialized congenital heart surgery teams, carrying out multicenter and multidisciplinary collaboration project in clinical and basic science research; (2) surgeons participating in research of surgery instrument, promoting the CHD surgery instrument localization and synchronization with adult surgery high-end instruments; (3) National patients with CHD data registry and clinical sample library establishment: surgery technique regionalization, and single category specialization for independent hospitals; (4) application of new techniques will promote rapid internationalization: using academic social media for easy data and knowledge sharing; introduction of robot and endoscopic surgery for minimal invasive therapy; (5) application of three-dimensional imaging and virtual reality techniques for operation under imaging.

\section{CONCLUSIONS}

Though with rapid progress, pediatric cardiac specialty in China still has no outstanding innovations and now is still chasing the steps of international peers. Advancing innovative surgery procedures requires the contributions of surgeons of one generation after another. Public awareness to CHD and surgeries is still lacking. Knowledge about prenatal diagnosis for CHD and intervention should be educated in pregnant women and the public. Due to the large population base, qualified fetal echocardiography doctors are far out of reach in China. There are still some regions not covered by CHD diagnosis and treatment network. So the network should be improved and initiate more pediatric cardiac centers. The government should increase the financial input in pediatric cardiac surgery and gradually increase the reimbursement rate of government-leading medical insurance for CHD. We should train more experience pediatric cardiac surgeons to grasp all complex techniques for improving patient care. There is still a large gap in recovery of patients with CHD after surgery between China and world leading countries. We should highlight the long-term recovery of patients with CHD after surgery including mental health and quality of life.

Contributors XM, DY and QS wrote the paper and the approved the final version as submitted.

Funding This study was supported by National Key Research and Development Program of China (2017YFC1308105 and 2016YFC1101001), and Zhejiang Provincial Program for the Cultivation of High Level Innovative Health Talents to QS.

Competing interests None declared.

Patient consent for publication Not required.

Provenance and peer review Commissioned; internally peer reviewed.

Open access This is an open access article distributed in accordance with the Creative Commons Attribution Non Commercial (CC BY-NC 4.0) license, which permits others to distribute, remix, adapt, build upon this work non-commercially, and license their derivative works on different terms, provided the original work is properly cited, appropriate credit is given, any changes made indicated, and the use is non-commercial. See: http://creativecommons.org/licenses/by-nc/4.0/.

\section{REFERENCES}

1. Thoracic and Cardiovascular Surgery Branch of Chinese Medical Association, The Thoracic and Cardiovascular Surgery Group of Pediatric Surgery Branch of Chinese Medical Association, National Society of Congenital Heart Disease. National consensus in China on perinatal diagnosis, evaluation and clinical disposal of cardiac birth defects. Chin J Pediatr Surg 2018;39:163-70. 
2. Population data. National data from national Bureau of statistics of China, 2016. Available: http://data.stats.gov.cn/easyquery.htm?cn= $\mathrm{C} 01 \& \mathrm{zb}=\mathrm{A} 0301 \& \mathrm{sj}=2016$ [Accessed 31 Aug 2018].
3. Hei FL, Zhu DM, Hou XT, et al. Chinese white paper on heart surgery and extracorporeal circulation data 2016. Chin J ECC 2017;15:65-7. 